\title{
Corrected QT Interval in Children With Brain Death
}

\author{
Jennifer Plymale $\cdot$ Jeanny Park • \\ JoAnne Natale $\cdot$ Anita Moon-Grady
}

Received: 30 March 2010/Accepted: 26 July 2010/Published online: 20 August 2010

(c) The Author(s) 2010. This article is published with open access at Springerlink.com

\begin{abstract}
Prolongation of the QT interval is a well-documented finding in adults with severe brain injury. However, QT prolongation has not been well documented in the pediatric population with brain injury. Our objective was to determine the range of QT intervals in children with the diagnosis of brain death, hypothesizing that the QT interval corrected for heart rate (QTc) is longer in this population than in a normal population. All previously healthy children $(<18$ years) dying in our hospital from 1995 to 2007 with a diagnosis of brain death and at least one electrocardiogram (ECG) with normal anatomy by echocardiogram were included. Admission details, past medical and family history, demographic data, and laboratory data were collected. The QT and preceding RR intervals from three sinus beats on a standard 12-lead ECG were measured. The QTc was calculated with the Bazett method, and the values were averaged. Thirty-seven patients met inclusion criteria. Five had event histories concerning for possible underlying rhythm disturbances; data analysis was performed with and
\end{abstract}

J. Plymale ( $\square)$

Herma Heart Center, Children's Hospital of Wisconsin,

Milwaukee, WI 53226, USA

e-mail: jplymale@chw.org

J. Park

Department of Pediatric Cardiology, UC Davis Medical Center,

University of California, Davis, Sacramento, CA 95817, USA

J. Natale

Pediatric Intensive Care Unit, UC Davis Medical Center,

University of California, Davis, Sacramento, CA 95817, USA

A. Moon-Grady

Pediatric Cardiology Division, University of California,

San Francisco, San Francisco, CA 94143, USA without these patients. The QTc data were normally distributed. The mean (SD) QTc for the entire cohort was 452 (61) ms. Excluding the five patients, it was 449 (62) ms. On multivariate analysis, sex (QTc female $<$ male) and hypokalemia were associated with QTc prolongation. QTc in children with brain death is normally distributed but significantly longer than QTc in normal children. Until rapid genetic testing for channelopathies is universally available, our findings suggest that potential pediatric cardiac donors with isolated prolongation of the QTc in this setting may be acceptable in the absence of other exclusionary criteria.

Keywords Brain death - Brain injury . Electrocardiographic changes · Long QT

\section{Introduction}

Electrocardiographic changes in brain injury have been well documented in the adult literature. An early study published in 1961 of 164 primarily adult patients with head injury found that the more common electrocardiographic abnormalities in head injury were a prolonged QT interval corrected for heart rate (QTc) and increased P-wave voltage [10]. Another early study addressing the pattern of electrocardiographic changes and the influence of cerebral regulation on heart activity was published in 1975 and reported prolongation of the QT interval in 21 of 28 patients with brain death [5]. This observation was extended to other neurologic injuries, including stroke. Although electrocardiographic changes are now a well-described entity in adults with stroke $[3,6,11,17]$, only small studies and case reports involving children have been previously published $[4,12,15,16]$. 
Etiologies for these electrocardiographic changes include sympathetic stimulation leading to tachycardia, increased $\mathrm{P}$ waves, short PR interval, increased QT interval, ST segment depression, and shallow T waves $[4,5,8,15]$. The autonomic nervous system (ANS) is an important modulator of ventricular repolarization, and the dysfunction that is seen in the ANS appears to be proportional to the degree of head injury [8]. In addition, cardiovagal autonomic function is known to decrease with age, partly due to gradual stiffening of the arterial wall, leading to impaired baroreflex function. A study of cardiovagal autonomic function in children found that neural autonomic mechanisms mature in children and attain peak level at adolescence. This maturational difference between children and adults may lead to different electrocardiographic changes in children versus adults with brain injury [13]. Further, electrolyte disturbances and many types of electrocardiographic changes are seen in adults with subarachnoid hemorrhage [3-5, 15, 17]. Of particular concern is the prolongation of QT interval often seen in patients who develop ventricular arrhythmias and eventual torsades de pointes. Patients with severe head injury are at high risk for electrolyte disturbances, including hypocalcemia, hypokalemia, and hypomagnesemia, which can be associated with QT interval prolongation and resulting arrhythmias. A dilemma occurs, however, in the evaluation of victims of both traumatic and ischemic injuries that lead to brain death. Pre-existing prolongation of the QT interval leading to arrhythmia may have been the cause of the injury and not simply an effect. Therefore, when evaluating potential cardiac organ donors, or when trying to determine the cause of death, the finding of a prolonged QT on electrocardiogram (ECG) may be difficult to interpret.

The aim of our study is to specifically evaluate the range of QT intervals of children with the diagnosis of brain death. Our hypothesis is that compared with the normal well pediatric population, QTc will be significantly longer in children with severe brain injury before or at the time of brain death.

\section{Materials and Methods}

\section{Study Population}

The University of California, Davis, Institutional Review Board gave approval and exemption for our study. All patients $<18$ years old who were hospitalized between 1992 and 2007 in the University of California, Davis, Pediatric Intensive Care Unit with (1) a diagnosis consistent with brain death either by International Classification of Disease, Ninth Revision, code 348.8 (condition of brain not else classified), 348.1 (anoxic brain injury), 780.01 (coma), 852.05-.25 (trauma subarachnoid hemorrhage), or charted diagnosis of "brain death" and (2) at least one 12-lead ECG obtained during hospitalization were evaluated for inclusion. Patients with significant pre-existing conditions (malignancy, cerebral palsy, chronic lung disease, congenital heart disease, acquired heart disease, or known rhythm abnormality) or comorbidities (infection) and those receiving medications known to prolong QTc were excluded. All charts fitting these criteria were available for review. All patients fitting these criteria were included, except one patient who died from a tricyclic antidepressant overdose.

\section{Data Collection}

A pediatrician extracted clinical data from the medical record. Reviewed records included admission history, consultation reports from pediatric neurologists and/or pediatric cardiologists, and preadmission medications. Brain injury was classified as traumatic, ischemic, or mixed based on progress notes, neuroimaging reports, discharge summary, and autopsy findings. Echocardiogram function was assessed by a pediatric echocardiographer as normal versus abnormal, with "normal" representing \%FS (shortening fraction) $\geq 95$ th percentile for age in the absence of significant valve regurgitation. If FS\% was borderline, then presence or absence of inotropic support was also taken into account in describing function as "normal" versus "abnormal." An evaluation of available information in the patient chart regarding family history and previous medical history that might suggest inclusion and/or exclusion of a diagnosis of (genetic) long QT syndrome (LQTS) was performed by a pediatric cardiologist (A. M.-G.), who was blinded to the ECG results. Electrolyte measurements from serum or whole blood included sodium, potassium, calcium, phosphorous, and magnesium. $\mathrm{pH}$ was measured by either venous or arterial blood gas. Axillary or rectal temperature recorded was correlated with timing of electrolyte measurements.

\section{Electrocardiograms}

The earliest available ECG result after admission was preferentially used. Rarely, multiple ECGs were performed. When more than one ECG was performed, the ECG completed in closest proximity to the time of the patient's most normal electrolyte and acid-base status was chosen. QT and RR interval measurements were recorded from each ECG by a single pediatric cardiologist/electrophysiologist (J. P.), who was blinded to clinical data. Lead II was used preferentially for QT measurements; however, lead V5 (another acceptable lead for QT measurement) was used in two cases due to excessive artifact. The QTc was calculated according to the Bazett formula (QT interval 
divided by the square root of the preceding RR interval). Three separate measurements by the same pediatric cardiologist/electrophysiologist were completed, and the mean of these was recorded as the QTc value for all analyses. QTc measurements were repeated on a subset of 30 patients by a second pediatric cardiologist (A. M.-G.) for evaluation of interobserver agreement (with $80 \%$ power). A previously published average QTc of healthy children of 399 ms (SD 20) [19] was used for comparison purposes.

\section{Statistical Analysis}

Means and SDs are reported for $\mathrm{pH}$, temperature, and electrolyte values obtained closest to the time of ECG analysis. Analysis of variance (ANOVA) was used to assess interobserver agreement in QTc between two pediatric cardiologists (A. M.-G. and J. P.). Univariate linear regression models were used to evaluate for correlation between QTc and electrolytes, $\mathrm{pH}$, temperature, sex, brain injury type, and ventricular function. Significant independent variables were entered into a multivariate regression model predicting QTc duration. Normality of distribution was assessed by construction of histograms and Q-Q plots (Fig. 1). We considered $p<0.05$ to represent statistical significance. Statistical programs used include R Development Core Team (2008; R: A language and environment for statistical computing. R Foundation for Statistical Computing, Vienna, Austria. ISBN 3-900051-07-0; http:// www.R-project.org) and Stata version 9 (Copyright 19962010 StataCorp, LP, College Station, TX, USA).

\section{Results}

Thirty-seven patients met all inclusion criteria. Demographic data, brain injury type, and ventricular function, as well as electrolyte, $\mathrm{pH}$, and temperature data as a means (SDs), are listed in Table 1.

Twenty-three (62\%) patients underwent ECG within $24 \mathrm{~h}$ of admission to the intensive care unit. Six $(16 \%)$ ECGs were performed within $48 \mathrm{~h}$, and the remaining 8 (22\%) ECGTs were performed after $48 \mathrm{~h}$ of admission. The QTc measurements for the cohort were normally distributed (Fig. 1a) with a mean (SD) of 452 (61) ms, which differed significantly versus published average QTc in healthy children of $399(20) \mathrm{ms}(p<0.001)$ [19]. Figure $1 \mathrm{~b}$ is a Q-Q plot (a scatter plot of the quantile of the observed data and the quantile of a normal distribution). Because $\mathrm{y}=\mathrm{x}$, the data are distributed normally, and our analysis is reasonable.

On univariate analysis of all 37 patients, lower potassium levels $(p=0.005)$, higher calcium $(p=0.008)$, and male sex $(p=0.001)$ were associated with increased QTc duration (Fig. 2). Tendencies toward higher QTc were seen with lower temperatures and with ischemic brain injury type, but this did not reach statistical significance. Age, abnormal echocardiographic function, sodium, and $\mathrm{pH}$ had no significant correlation with QTc (Table 2). Multivariate analysis found lower potassium level $(p=0.006)$ and male sex $(p=0.003)$ to be significantly associated with increased QTc (Table 3).

A chart review of the admission history and physical, past medical history, and family history showed a concern for possible congenital long QT syndrome in five patients (history of seizures, a witnessed sudden collapse with exercise, sudden infant death syndrome, or the patient was found down unresponsive). In analyses that excluded these patients, QTc remained normally distributed with a mean of 449 (SD 62) ms, and the significant effect of potassium level and sex on QTc duration remained unchanged. Exclusion of these five patients from multivariate regression analysis did not affect the findings (Table 3).

Interobserver reliability was determined for the difference between measurements by two different cardiologists (A. M.-G. and J. P.). There was strong agreement between the two observers by ANOVA, with an intraclass correlation of 0.967 with a $95 \%$ confidence interval of $0.943,0.990$.

\section{Discussion}

We show that in otherwise previously healthy children admitted to an intensive care unit with the diagnosis of brain death, the average QTc was significantly prolonged at 452 (61) ms versus a published average QTc in healthy children of 399 (20) ms $(p<0.001)$ [19]. We found male sex and hypokalemia to be independent risk factors for QTc prolongation. Purely ischemic brain injury led to a
Fig. 1 Histograms and Q-Q plots for evaluation of data distribution. a Measured QTc in six bins with a resultant normal distribution for all patients. b Q-Q plot confirming normality of the data
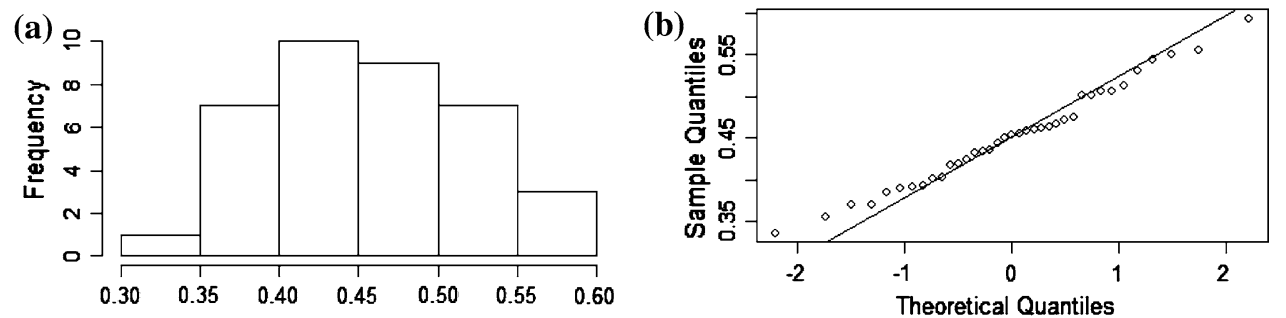
Table 1 Clinical, echocardiographic, and biochemical data at time of ECG in 37 children with brain death

\begin{tabular}{lccc}
\hline Variable & $\begin{array}{l}\text { Number } \\
(\%)\end{array}$ & Value (SD) & $\begin{array}{l}\text { QTc (SD) } \\
\text { ms }\end{array}$ \\
\hline Age (years) & 37 & $6.21(5.4)$ [range 0-17] & $452(61)$ \\
Gender & & & $468(55)^{*}$ \\
Male & $29(78)$ & & $394(45)$ \\
Female & $8(22)$ & & \\
Ethnicity & & & \\
African American & $2(5)$ & & $481(70)$ \\
Hispanic & $11(30)$ & & $456(52)$ \\
White & $18(49)$ & & $407(48)$ \\
Unknown & $6(16)$ & & \\
Injury type & & & $444(62)$ \\
Ischemic & 10 & & $466(61)$ \\
Traumatic & 19 & & \\
Mixed & 8 & & \\
Ventricular function & \\
Normal & 25 & & \\
Abnormal & 12 & & \\
Sodium (mEq/l) & 37 & $148.8(10.4)$ & \\
Potassium (mEq/l) & 37 & $3.8(0.9)$ & \\
pH & 37 & $7.33(0.13)$ & $(1.0)$ \\
Calcium (mg/dl) & 32 & 8.7 & \\
Magnesium (mg/dl) & 34 & $2.0(0.5)$ & \\
Phosphorus (mg/dl) & 35 & $4.6(3.0)$ & \\
Temperature ( $\left.{ }^{\circ} \mathrm{C}\right)$ & 36 & $36.1(1.7)$ & \\
\hline
\end{tabular}

$S D$ standard deviation

$* p<0.05$

more profound QTc prolongation. The effect of brain injury on QTc tended to be enhanced by conditions of relative hypothermia, higher sodium level, and higher calcium level. Excluding five children with histories concerning for possible LQTS, we still found the remaining group to have significantly prolonged average QTc and the same associations.

Possible etiologies for prolongation of QT in acute brain injury have been hypothesized in adults. Goldstein et al. found evidence of uncoupling of the autonomic and cardiovascular systems during acute brain injury and brain death caused by decreased heart beat oscillations and decreased barosensitivity from the injury. The degree of coupling was directly related to the degree of insult [9]. Our study found the more severe the brain injury (ischemic injury), the longer the QTc. This could be explained by more severe injuries correlating with greater uncoupling of the autonomic and cardiovascular systems. Thus, our study findings support the findings of Goldstein [9]. Another etiology for the prolongation of QT in cerebrogenic cardiovascular disturbances includes sympathetic stimulation leading to multiple changes on ECG. These include not only increased QT interval but also tachycardia, increased $\mathrm{P}$ waves, short PR interval, decreased or increased ST segments, flat or inverted T waves, peaked T waves, U waves, Q waves, and increased QRS amplitude [1]. The cardiospecific isoenzyme creatine kinase MB has also been found to be increased in patients having ECG changes with cerebrogenic changes. This has been reported in adults but not in published case reports of children [1, 12]. Our study supports the increased QT interval in children with brain injury even though we did not measure creatine kinase MB.

Our findings are in agreement with studies in adult populations with brain injury [3, 6, 13]. In 2003, Fukui reported a study, comprising 100 adult patients with subarachnoid hemorrhage, in which patients were found to have an average QTc of $466 \pm 46$ on 12-lead ECG performed within $24 \mathrm{~h}$ of injury. On multivariate analysis, a significant relation between QTc and sex, potassium, calcium, and glucose levels was found; female sex and low potassium level were found to be independent risk factors for prolonged QTc in this group with subarachnoid hemorrhage [6]. Although we saw a similar association with potassium level, we found male sex to be associated with longer QTc in our population. This may be due in part to the small number of girls included in our study, but it may also reflect a maturational effect or perhaps the inclusion of patients with all types of brain injury and not just hemorrhage $[13,21]$. In addition, although natural history suggests that before puberty, boys are more at risk for long QT-induced cardiac arrest, studies have shown that girls become more at risk as they grow older [14, 21]. This also supports the difference in our article versus Fukui [6].

Published case reports in children include several with severe head trauma exhibiting ischemic ECG changes and other reports of QT interval prolongation after minor head trauma [4, 12]. In a larger study, Ozdemir recently reported a study of head trauma and effect on QT interval and QT dispersion in children. They reported on 43 children, and those with severe head trauma and intracranial hemorrhage in particular ( $n=16$ patients) exhibited prolonged QT intervals (QTc $>440$ in 37 vs. 6\% controls). These changes were inversely proportional to Glasgow Coma Scale score, serum calcium levels, and potassium levels; the majority of these patients, however, did not satisfy criteria for brain death. They also found QT dispersion to be longer in this group [16]. The present study extends these observations to patients with brain death, further strengthening the association of severe injury with QT prolongation; however, we did not assess QT dispersion, which may correlate better with the risk of ventricular arrhythmias in both children and adults [11, 16]. 

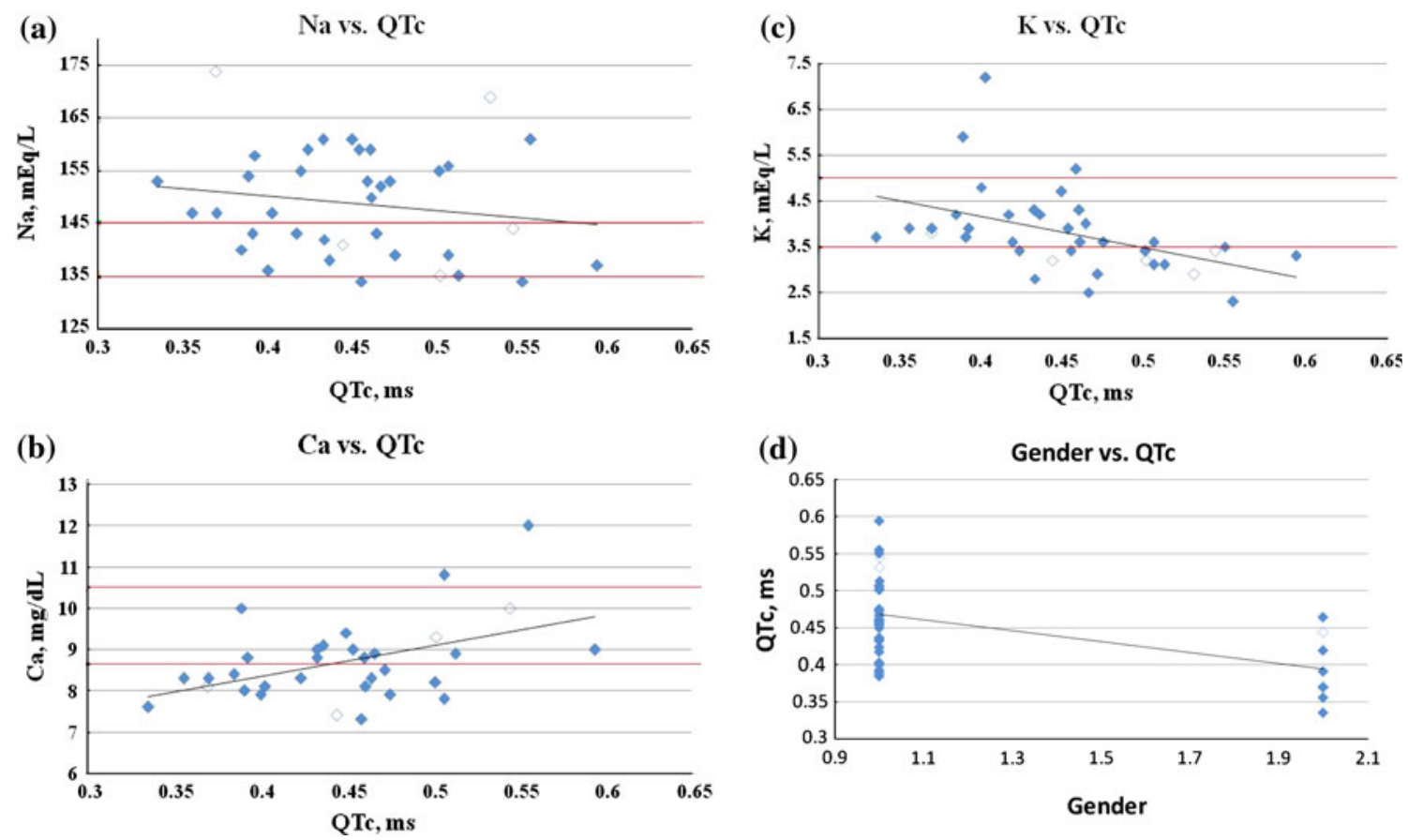

Fig. 2 Scatter plots for significant variates in univariate analysis. a Na vs. QTc, b Ca vs. QTc, c K vs. QTc, d Gender vs. QTc. $1=$ male; $2=$ female. Open symbols represent five patients with clinical history suggestive of possible LQTS

Table 2 Univariate regression analysis of factors associated with QTc prolongation in children with brain death

\begin{tabular}{lcc}
\hline Variable & Coefficient $(95 \% \mathrm{CI})$ & $p$ \\
& $N=37$ & \\
\hline Female sex & $-0.074(-0.117$ to -0.031$)$ & 0.001 \\
Potassium & $-0.03(-0.05$ to -0.01$)$ & 0.005 \\
Calcium & $0.029(-0.008$ to 0.049$)$ & 0.008 \\
\hline
\end{tabular}

CI confidence interval

$p<0.05$ is statistically significant

Our study is limited by several factors. First, our study population was small and predominately male (78\%). This is likely due to the fact that trauma or accident is the most common cause of morbidity and mortality in children, and that statement is particularly true for male children. The small number of girls may have limited our ability to accurately represent sex differences, particularly when analyzing injury subtypes. Second, no posttransplant QTc information was available for donated organs (to evaluate for normalization), nor was genetic testing available to ensure that the patients did not have an inherited form of long QT syndrome. However, given the population incidence of familial LQTS of approximately 1:3000 to 1:5000 $[2,7,18,20]$, it is unlikely that a significant number of our patients carried a genetic mutation. Third, our study is retrospective and thus may be subject to information bias. All investigators were aware of the diagnosis of brain death in the patients and of the hypothesis. QTc measurement data were, however, reproducible with little interobserver variability.

There are two major implications of our findings. First, in determining the cause of brain death in children who are apparent victims of accidental trauma or drowning, or who

Table 3 Multivariate analysis of factors associated with QTc prolongation in children with brain death

\begin{tabular}{lllll}
\hline Variable & Coefficient $(95 \% \mathrm{CI})$ & $p$ & $\begin{array}{l}\text { Coefficient }(95 \% \text { CI }) \\
N=32^{\mathrm{a}}\end{array}$ \\
\hline Female sex & $-0.068(-0.11$ to -0.03$)$ & 0.003 & $-0.073(-0.12$ to -0.03$)$ & 0.004 \\
Potassium & $-0.026(-0.04$ to 0.01$)$ & 0.056 & $-0.028(-0.05$ to -0.01$)$ & 0.008 \\
Calcium & $0.011(-0.01$ to 0.03$)$ & 0.267 & $0.007(-0.01$ to 0.03$)$ & 0.504 \\
\hline
\end{tabular}

CI confidence interval

$p<0.05$ is statistically significant

${ }^{\text {a }}$ Five patients with clinical history suggestive of possible LQTS were excluded from analysis 
are found unresponsive, an ECG is often part of the evaluation. Our results suggest that even a substantial prolongation of the QTc may in fact be secondary to brain injury (by an unknown mechanism) and not a primary cause of the patient's death. Second, when considering children with brain death as possible organ donors, an ECG is often performed as a screening tool before considering the heart for donation. Whether or not these children have congenital long QTc versus brain injury-related QTc prolongation may be difficult to determine in the absence of rapid genetic testing. Until rapid genetic testing for channelopathies is universally available, our findings suggest that potential pediatric cardiac donors with isolated prolongation of the QTc in this setting may be acceptable in the absence of other exclusionary criteria.

Acknowledgments Support for two of the authors (J. M. Plymale and A. M.-G.) was provided in part by a grant for the Children's Miracle Network. Statistical support was made possible by Grant No. UL1 RR024146 from the National Center for Research Resources (NCRR), a component of the National Institutes of Health (NIH), and NIH Roadmap for Medical Research. Its contents are solely the responsibility of the authors and do not necessarily represent the official view of NCRR or NIH. Information on Re-engineering the Clinical Research Enterprise can be obtained from http://nihroadmap. nih.gov/clinicalresearch/overview-translational.asp.

Open Access This article is distributed under the terms of the Creative Commons Attribution Noncommercial License which permits any noncommercial use, distribution, and reproduction in any medium, provided the original author(s) and source are credited.

\section{References}

1. Cheung RT, Hachinski V (2000) The insula and cerebrogenic sudden death. Arch Neurol 57:1685-1688

2. Chiang CE, Roden DM (2000) The long QT syndromes: genetic basis and clinical implications. J Am Coll Cardiol 36:1-12

3. Collier BR, Miller SL et al (2004) Traumatic subarachnoid hemorrhage and QTc prolongation. J Neurosurg Anesthesiol 16:196-200
4. Dash M, Bithal PK et al (2003) ECG changes in pediatric patients with severe head injury. J Neurosurg Anesthesiol 15:270-273

5. Drory Y, Ouaknine G et al (1975) Electrocardiographic findings in brain death: Description and presumed mechanism. Chest $67: 425-432$

6. Fukui S, Katoh $\mathrm{H}$ et al (2003) Multivariate analysis of risk factors for QT prolongation following subarachnoid hemorrhage. Crit Care 7:R7-R12

7. Goldenburg I, Moss A (2008) Long QT syndrome. J Am Coll Cardiol 51:2291-2300

8. Goldstein B, DeKing D et al (1993) Autonomic cardiovascular state after severe brain injury and brain death in children. Crit Care Med 21:228-233

9. Goldstein B, Toweill D et al (1998) Uncoupling of the autonomic and cardiovascular systems in acute brain injury. Am J Physiol 275(4 Pt 2):R1287-R1292

10. Hersch C (1961) Electrocardiographic changes in head injuries. Circulation 23:853-860

11. Huang CH, Chen WJ et al (2004) QTe dispersion as a prognostic factor in intracerebral hemorrhage. Am J Emerg Med 22:141-144

12. Koturoglu G, Kurugol Z et al (2006) QT prolongation and minor head trauma: a report of two cases. Minerva Pediatr 58:575-577

13. Lenard Z, Studinger P et al (2004) Maturation of cardiovagal autonomic function from childhood to young adult age. Circulation 110:2307-2312

14. Locati E, Zareba W et al (1998) Age- and sex-related differences in clinical manifestations in patients with congenital long QT syndrome: findings from the International LQTS Registry. Circulation 97:2237-2244

15. McLeod AA, Neil-Dwyer G et al (1982) Cardiac sequelae of acute head injury. Br Heart J 47:221-226

16. Ozdemir D, Ozdemir N et al (2005) QTc dispersion in children with severe head trauma. Pediatr Emerg Care 21(10):658-661

17. Perron AD, Brady WJ (2000) Electrocardiographic manifestations of CNS events. Am J Emerg Med 18:715-720

18. Schwartz PJ, Priori SG, Napolitano C (2003) How really rare are rare diseases? J Cardiovasc Electrophysiol 14:1120-1121

19. Tutar HE, Ocal B et al (1998) Dispersion of QT and QTc interval in healthy children, and effects of sinus arrhythmia on QT dispersion. Heart 80:77-79

20. Vincent GM, Timothy KW et al (1992) The spectrum of symptoms and QT intervals in carriers of the gene for the long-QT syndrome. N Engl J Med 327:846-852

21. Zareba W, Moss AJ et al (2003) Modulating effects of age and gender on the clinical course of long QT syndrome by genotype. J Am Coll Cardiol 42:103-109 